\title{
CALIDAD DE UN CURSO VIRTUAL DE E-LEARNING EN CIENCIAS DE LA SALUD
}

\section{QUALITY OF AN E-LEARNING VIRTUAL COURSE IN HEALTH SCIENCES}

\author{
Judith I Francisco Pérez ${ }^{1}$ jifrancisco@utpl.edu.ec \\ Judith J. Maldonado-Rivera² jjmaldonado@utpl.edu.ec \\ Manuel Ramón Blanco ${ }^{3}$ mrblanco@utpl.edu.ec \\ Universidad Técnica Particular de Loja, Ecuador
}

\author{
Volumen 8, Número 1 \\ 30 de mayo de 2017 \\ pp. $94-120$
}

Recibido: 13 de diciembre de 2016

Aprobado: 30 de abril de 2017

\footnotetext{
${ }^{1}$ Licenciada en Enfermería (Universidad de Carabobo, Venezuela), Magíster en Educación, Mención Planificación Curricular (Universidad de Carabobo, Venezuela), Doctora en Tecnología Instruccional y Educación a Distancia (NOVA Southeastern University, USA). Algunas de las investigaciones publicadas son: "Diseño de una Especialización en E-learning Enfocada por Competencias, Experiencias Significativas de Aprendizaje de un Grupo de Profesionales de Enfermería Cursantes del Diplomado de Cuidados Intensivos Pediátrico y Neonatal, ante el B-learning y Guía Para el Diseño Instruccional". Actualmente desarrolla su carrera académica como coordinadora de la Maestría "Gerencia en Salud para el Desarrollo Local", en la Universidad Técnica Particular de Loja, Ecuador. Email: jifrancisco@utpl.edu.ec

2 Docente Universidad Técnica Particular de Loja (UTPL) Ecuador. Profesora titular de la UTPL. Departamento de Ciencias de la Educación. Sección: Organización y Planificación Educativa en la Universidad Técnica Particular de Loja (UTPL). Unidad de Evaluación Institucional. Ecuador. E-mail: jjmaldonado@utpl.edu.ec

${ }^{3}$ Docente Jubilado del Decanato de Ciencias de la Salud. Universidad Centroccidental Lisandro Alvarado (UCLA) Venezuela. Médico Cirujano (UCLA), Internista (Hospital Universitario Dr. Luis Gómez López, Venezuela), Magíster en Salud Pública (UCLA). Docente e Investigador agregado, jubilado de la UCLA. Coordinador de Producción de Medios Educativos del Sistema de Educación a Distancia de la UCLA. Director Técnico de Apoyo Académico en la misma Universidad. Actualmente desarrolla su carrera académica como docente investigador de la titulación de Medicina de la Universidad Técnica Particular de Loja, Ecuador. Email:mrblanco@utpl.edu.ec
}

Calidad de un curso virtual de e-learning en ciencias de la salud Judith I Francisco Pérez, Judith J. Maldonado-Rivera, Manuel Ramón Blanco

DOI: http://dx.doi.org/10.22458/caes.v8i1.1774

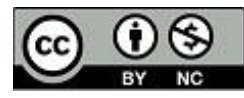

Artículo protegido por licencia Creative Commons 


\section{Resumen}

Se realizó una investigación descriptiva con el objetivo de evaluar la calidad de un curso virtual de formación docente, usando el Modelo de Evaluación de Cursos Virtuales de Formación Continua del Instituto Latinoamericano de Calidad en Educación a Distancia (CALED). Mediante la aplicación de una lista de verificación y cuatro cuestionarios, se analizaron 72 indicadores de las áreas: calidad tecnológica, formación, diseño instruccional y servicios y soporte. Los resultados indican cumplimiento parcial de los estándares de calidad. Las áreas de mayor cumplimiento en orden decreciente fueron: formación, diseño instruccional y servicios y soporte. En el área de calidad tecnológica se cumple con la mayoría de los requerimientos y en algunos casos no se encontraron registros requeridos como evidencias de la evaluación.

Palabras Clave: Educación a distancia; evaluación de la educación; calidad de la educación; formación de docentes.

\section{Abstract}

A descriptive study was conducted in order to evaluate the quality of a virtual course on teacher training, using the Evaluation Model of the Virtual Continuous Training Courses of the Latin American Institute for Quality in Distance Education (CALED, in Spanish). By applying a checklist and four questionnaires, there were analyzed 72 indicators for the following areas: Technological quality, training, instructional design and services and support. The results indicate a partial accomplishment of the quality standards. The areas of greatest fulfillment in descending order were training, instructional design and services and support. In the area of technological quality most of the requirements were met, but in many cases, no required records were found as evidence at the time of the assessment.

Keywords: Distance education; educational evaluation; educational quality; teacher education. 


\section{INTRODUCCIÓN}

La educación a distancia $(\mathrm{EaD})$ es un sistema de comunicación bidireccional de carácter masivo que propicia el aprendizaje independiente y flexible de los estudiantes, sustituyendo la interacción presencial del profesor y el alumno por una acción sistemática de diversos recursos didácticos, con apoyo institucional (García Aretio, 1994). Esta modalidad educativa goza hoy en día de una extensa aplicabilidad en la educación superior (García Aretio, 2016), y su principal característica es la implementación de un diálogo didáctico mediado a través de recursos de aprendizaje en formato impreso o digital (García Aretio, 1994).

En ese sentido, la EaD se viabiliza mediante equipos docentes multidisciplinarios, los cuales diseñan y producen materiales didácticos que promueven los diferentes tipos de diálogo, generando tanto aprendizaje individual como cooperativo y colaborativo (García Aretio, 2012). Estos equipos garantizan la implementación de canales de comunicación para la entrega de contenidos estructurados, propiciando el autoestudio, la mediación y la interacción.

Según Cabero-Almenara (2016), la EaD ha experimentado un salto cualitativo significativo en las últimas décadas, pasando de ser considerada una educación de segundo orden con una imagen poco prestigiosa, a ser contemplada como una educación con altas potencialidades. En este escenario han tenido un papel determinante las tecnologías de información que han impactado el desarrollo de la sociedad en general y de la educación en particular.

Es así como surge el e-learning, una evolución lógica de la EaD soportada en sistemas digitales, cuyo desarrollo y avance ha propiciado un acortamiento de las distancias, asegurando al menos, la misma calidad de la educación presencial (García Aretio, 2013). La

Calidad de un curso virtual de e-learning en ciencias de la salud Judith I Francisco Pérez, Judith J. Maldonado-Rivera, Manuel Ramón Blanco

DOI: http://dx.doi.org/10.22458/caes.v8i1.1774

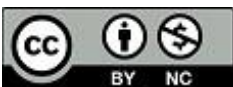

Artículo protegido por licencia Creative Commons 
eficacia de esta modalidad depende del uso de recursos tecnológicos, de la formación de los profesores, la participación de los estudiantes y de la calidad de sus diseños didácticos (García Aretio, 2016), estructurados pedagógicamente en entornos virtuales de aprendizaje (EVA). Estos entornos se comportan como espacios de interacción sociocultural, generados, mediados y potenciados por las TIC, donde los sujetos en formación socializan y se apropian de nuevos conocimientos, habilidades, actitudes, valores, formas de comportamiento y experiencias, sustentados en un modelo pedagógico (García, Guerrero y Granados, 2015).

Los EVA reúnen en un mismo espacio todas las actividades de interacción, recursos multiformato, experiencias, instrucciones y aplicaciones, que contribuyen de manera coherente a los resultados de aprendizaje planificados en diseño instruccional. Así, se convierten en plataformas que posibilitan la disposición en red, de todo aquello que facilita la autogestión del conocimiento y la interacción con los contenidos, los asesores y los estudiantes, cuyo diseño debe estar vinculado a la calidad de la formación (Ávila y Barragán, 2015). Tomando en cuenta que las tecnologías más avanzadas, bien utilizadas, pueden propiciar una "conversación didáctica guiada", con grandes posibilidades de interacción (García Aretio, 2012).

Por lo antes expuesto, la implementación del e-learning debe cuidar aspectos de carácter pedagógico, tecnológico, metodológico, organizacional y social, que en forma integrada permiten confrontar las dificultades de los cursos en modalidad virtual (García, Guerrero y Granados, 2015). Es decir, asumir el reto de demostrar cohesión entre calidad tecnológica y calidad pedagógica (Ardila-Rodríguez, 2011), integrando eficientemente las tecnologías de información en espacios de aprendizaje que promuevan la autonomía, la colaboración y la conexión con la realidad (Núñez, 2017).

Calidad de un curso virtual de e-learning en ciencias de la salud Judith I Francisco Pérez, Judith J. Maldonado-Rivera, Manuel Ramón Blanco

DOI: http://dx.doi.org/10.22458/caes.v8i1.1774

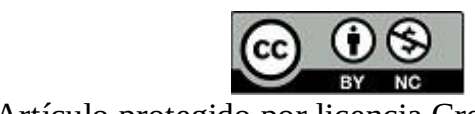

Artículo protegido por licencia Creative Commons 
En lo tecnológico, la calidad de la educación virtual implica disponer de tecnologías pertinentes, actualizadas y adecuadas, las cuales deben ser contempladas en los presupuestos de inversión. Mientras que en lo pedagógico y metodológico se debe considerar la formación de los docentes como pilar fundamental para elevar la calidad de la educación superior en el marco del nuevo paradigma educativo de la sociedad de la información (Aguilar y Bautista, 2015).

La calidad es uno de los criterios más aludidos en las declaraciones filosóficas de las instituciones universitarias en todo el mundo, como expresión de su interés por alcanzar estándares que les permitan posicionarse como centros de reconocida eficiencia y elevado nivel de formación (Francisco, 2012). Uno de los referentes empleados para la evaluación de calidad de la educación a distancia es el modelo CALED, estructurado en base al Potencial Marco Regulador para la Oferta de Cursos Virtuales del "Centro Virtual para el Desarrollo de Estándares de Calidad para la Educación Superior a Distancia” (Rubio, M.J. et al. 2009). En este modelo los estándares de calidad y los indicadores adoptan las características propias del curso a evaluar, así como aquellas otras que contribuyen a su organización y gestión. Comprende 4 áreas, 16 subáreas, 26 estándares y 72 indicadores y propone una ponderación de 100 puntos desglosados en las áreas y subáreas correspondientes, descritas a continuación:

- Calidad Tecnológica: Se califica con un máximo de 30 puntos; evalúa disponibilidad tecnológica, rendimiento, capacidad, seguridad, privacidad, accesibilidad, usabilidad/navegabilidad y mantenimiento. El cumplimiento de estos indicadores, garantiza el correcto funcionamiento y desarrollo tecnológico de los cursos. 
- Formación: Se califica con 15 puntos; evalúa la disponibilidad y ejecución de los planes de formación pedagógica y técnica con la que deben contar los docentes y alumnos para afrontar su correspondiente rol.

- Diseño Instruccional. Se califica con 45 puntos; evalúa la estructura, diseño, contenidos y metodología del curso.

- Servicios y Soporte: Tiene una calificación de 10 puntos; evalúa la disponibilidad de servicios de información y de atención al estudiante para desarrollar normalmente sus actividades.

El modelo CALED surge con la misión de contribuir al mejoramiento en la enseñanza superior a distancia en todas las instituciones de América Latina y el Caribe, brindando asesoría principalmente en los procesos de autoevaluación y acreditación a las universidades y a los programas a distancia que así lo requieran, basados en criterios, indicadores y estándares comunes para la educación superior de la calidad. Por esta razón, en la presente investigación se aplicaron los estándares sugeridos por este modelo para evaluar la calidad de un curso de formación docente en el Decanato de Ciencias de la Salud de la UCLA.

El curso evaluado se ofrece en modalidad virtual desde el año 2007 con el propósito de capacitar a los docentes para emplear el entorno Moodle como apoyo en la enseñanza presencial. Sus resultados podrían servir a las autoridades de la institución, a los diseñadores y los responsables del Sistema de Educación a Distancia de la UCLA (SEDUCLA), para incorporar correctivos y fortalecer aspectos que redunden en la calidad de la formación. Conscientes que es necesario fortalecer la formación de los docentes, de manera que ellos aprendan a desarrollar sistemas de apoyo para la producción de entornos virtuales y materiales educativos de calidad (Cabero-Almenara, 2016).

Calidad de un curso virtual de e-learning en ciencias de la salud Judith I Francisco Pérez, Judith J. Maldonado-Rivera, Manuel Ramón Blanco

DOI: http://dx.doi.org/10.22458/caes.v8i1.1774

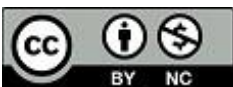




\section{METODOLOGÍA}

Tipo de Investigación.

Se trata de un estudio descriptivo, transversal. De acuerdo con Hernández, Fernández y Baptista (2014), los estudios descriptivos se ocupan de presentar los hechos tal como son observados, sin manipular las variables ni establecer entre ellas explicación o correlación. Asimismo, los estudios transversales son aquellos que se realizan en un tiempo determinado sin que se considere la evolución del fenómeno bajo estudio. En ese sentido, la investigación tuvo como propósito evaluar la calidad del curso virtual en un momento determinado, de acuerdo con el cumplimiento de los estándares establecidos en el modelo seleccionado, proceso basado en la observancia de evidencias que permitieron establecer la puntuación de cada subárea y sus indicadores, reflejado finalmente de manera sistemática en un informe de evaluación.

\section{Descripción del Objeto de Estudio.}

El curso virtual para la formación de docentes del Decanato de Ciencias de la Salud de la UCLA, denominado Docencia en Entornos Virtuales de Aprendizaje (DEVA), se oferta desde al año 2007 y se diseñó con la finalidad de ofrecer capacitación para la enseñanza en modalidad virtual a los profesores que requerían incorporar dentro de sus cursos presenciales, el uso de nuevas tecnologías de información y comunicación, en concordancia con lo establecido en las políticas académicas de la universidad. Se desarrolla en 12 semanas continuas que abarcan las siguientes unidades:

- Unidad I. Introducción al Sistema de Educación a Distancia de la UCLA (SEDUCLA).

- Unidad II. Marco Legal de la Educación a Distancia (EaD).

- Unidad III. Diseño Instruccional para la EaD.

- Unidad IV. Diseño de Materiales Didácticos.

- Unidad V. Diseño del Aula Virtual en Moodle.

Calidad de un curso virtual de e-learning en ciencias de la salud Judith I Francisco Pérez, Judith J. Maldonado-Rivera, Manuel Ramón Blanco

DOI: http://dx.doi.org/10.22458/caes.v8i1.1774 
Participantes.

Participaron el equipo tecnológico, el coordinador responsable, diez egresados y dos docentes del curso.

Técnicas e Instrumentos Utilizados.

Con base en la guía de evaluación para cursos virtuales de formación continua del instituto CALED, se organizaron los formularios para el registro de autoevaluación que se aplicaron a los informantes, adaptados de acuerdo a las diferentes áreas, subáreas, estándares e indicadores del modelo. La autorización para el uso de esta guía y la aplicación de los formularios fue obtenida en el marco de una pasantía realizada por la investigadora principal en la sede de este Instituto en la Universidad Técnica Particular de Loja, en la ciudad de Loja, Ecuador. Siendo la UCLA, una institución asociada al mismo, este proceso de autoevaluación se desarrolló en el marco de los acuerdos del convenio interinstitucional suscrito entre ambas universidades como parte de los procesos necesarios para elevar la calidad educativa de la UCLA en modalidad virtual.

En este sentido se aplicaron cuatro instrumentos, constituidos por preguntas de selección múltiple, cada una con un espacio para observaciones y propuestas de mejora. Asimismo, se elaboró un registro de observación, compuesto por aquellos indicadores de las diferentes áreas y subáreas que pueden ser evidenciados en el entorno virtual. Como técnicas de evaluación se aplicaron la observación directa y la encuesta.

\section{Procedimiento.}

Para alcanzar los objetivos del estudio se aplicaron las siguientes etapas:

- Planificación del proceso de autoevaluación, donde se determinó el cronograma de trabajo, responsables de cada proceso y presupuesto necesario.

Calidad de un curso virtual de e-learning en ciencias de la salud Judith I Francisco Pérez, Judith J. Maldonado-Rivera, Manuel Ramón Blanco DOI: http://dx.doi.org/10.22458/caes.v8i1.1774 
- Sensibilización, llevada a cabo mediante reuniones de información y concienciación para el personal docente, administrativo y de servicios, sobre el propósito y la importancia de la investigación con fines de mejoramiento del programa de formación.

- Diseño de técnicas e instrumentos, donde se construyeron cuatro cuestionarios para los informantes y un registro de observación para uso de los investigadores.

- Recopilación, organización y sistematización de información, la cual se realizó mediante el aporte del equipo de trabajo específico designado, así como del equipo de apoyo para tareas concretas (estadística, análisis de datos y comprobación de evidencias).

- Elaboración del informe final, para lo cual se utilizó el "Registro de Autoevaluación" que constituye el instrumento básico del mismo modelo y contiene información referente a: informantes calificados, fuentes de información, lugar en donde reposan los documentos de verificación, técnicas e instrumentos aplicados en el proceso, código de los documentos, valoración obtenida en cada área, criterios y estándares correspondientes, y propuestas de mejora.

En general, el procedimiento desarrollado implicó la participación de todos los involucrados en el proceso formativo, quienes aportaron información requerida para identificar el cumplimiento de los estándares, permitiendo orientar las conclusiones, recomendaciones y propuestas con miras a la búsqueda de calidad. 


\section{RESULTADOS}

\section{Área Tecnología}

Infraestructura Tecnológica.

Al inicio del curso virtual se aplica una prueba de análisis de la audiencia, que entre otros aspectos indaga acerca de la capacidad de acceso a internet de los participantes, así como, las necesidades de éstos en concordancia con los tipos de interacciones que se realizarán. Sin embargo, no se identifica la disponibilidad ni la naturaleza de los equipos y programas informáticos con que cuentan los participantes, ni se observa la velocidad de las conexiones a internet que ellos poseen. En cada departamento donde laboran los participantes, se dispone de equipos y herramientas tecnológicas, mas se desconoce si éstas son suficientes y adecuadas para el programa de formación. De acuerdo con la información descrita, la subárea "Infraestructura tecnológica" cumple con el 57\% de los estándares recomendados en el modelo CALED, con una valoración de 1.7 sobre 3.

Disponibilidad, Rendimiento y Capacidad

Se cuenta con un equipo que garantiza la estabilidad del suministro eléctrico. La capacidad de los equipos y sistemas informáticos es adecuada para el volumen de usuarios y transacciones estimadas. La memoria secundaria es suficiente para soportar la cantidad de datos generados, y la memoria primaria es suficiente para soportar la carga de procesamiento de información proyectada por cada curso. Sin embargo, no existen planes de contingencia ante problemas técnicos y de previsión de tolerancia a fallos. Esta documentación estaba en proceso de elaboración para el momento de la investigación. Según los estándares sugeridos en el modelo CALED, se cumple con $71,25 \%$, con una valoración de 4.3 sobre 6 puntos.

Calidad de un curso virtual de e-learning en ciencias de la salud Judith I Francisco Pérez, Judith J. Maldonado-Rivera, Manuel Ramón Blanco

DOI: http://dx.doi.org/10.22458/caes.v8i1.1774

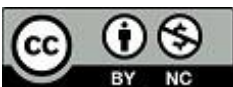


Seguridad y Privacidad

Existe un plan de seguridad para el acceso a las instalaciones y sistemas informáticos, y un equipo humano responsable de aplicar las políticas de seguimiento y respaldo. De acuerdo con la legislación vigente en materia de privacidad y custodia de los datos personales, el Manual de Procedimientos de la Dirección de Telecomunicaciones (UCLA, 2009a) establece que el intercambio de datos sensibles debe realizarse mediante conexiones encriptadas, no obstante, no hay registro de su cumplimiento.

Los sistemas de respaldo están robotizados mediante un programa de automatización (TCM) que realiza este proceso diariamente, sin embargo, no se encontró evidencia de los lineamientos y normas en relación a este proceso. De igual manera, no se cuenta con enlaces redundantes. De acuerdo con el modelo CALED, existe cierto avance en el cumplimiento de los estándares establecidos para esta sub área, que corresponde al $50 \%$; equivalente a una valoración de 3 puntos sobre 6 .

\section{Accesibilidad}

La accesibilidad está garantizada en el artículo 82 del Reglamento de EaD de la universidad (UCLA, 2009b), en donde se establece que el curso, antes de ser ofertado en el entorno formal del sistema, debe cumplir con una fase experimental, en donde se realizan entre otras, todas las pruebas de funcionamiento y penetrabilidad a los contenidos ofrecidos. Asimismo, antes de su implementación formal, todo curso debe evidenciar que su planificación esté basada en un modelo de diseño instruccional y se fundamente en un modelo pedagógico que oriente y direccione el proceso de formación que se desea implantar. Sin embargo, no se encontró el registro donde se haga constar, que efectivamente el curso fue migrado de un entorno a otro habiendo cumplido con todos los requerimientos.

Calidad de un curso virtual de e-learning en ciencias de la salud Judith I Francisco Pérez, Judith J. Maldonado-Rivera, Manuel Ramón Blanco

DOI: http://dx.doi.org/10.22458/caes.v8i1.1774

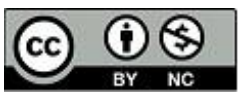

Artículo protegido por licencia Creative Commons 
Por otra parte, se pudo evidenciar que se han seleccionado los medios adaptados a las necesidades de enseñanza-aprendizaje y las tecnologías estándares y abiertas necesarias. Asimismo, que existe una organización adecuada de la información, del diseño en general y un uso correcto de etiquetas de marcaje. También se pudo identificar que el curso virtual fue evaluado y aprobado por la comisión académica de la UCLA, lo cual implica que su diseño fue considerado idóneo por todos los directores académicos de la universidad, aunque no se encontró registro que evidencie de manera pormenorizada cada uno de los aspectos evaluados y la calificación cualitativa y cuantitativa otorgada. Por otra parte, se constató la utilización de tecnologías estándares y abiertas y el uso de la plataforma Moodle, la cual cuenta con tecnologías de actualización y asistencia.

De acuerdo con el análisis realizado, los estándares de la presente subárea alcanzan $45 \%$ de cumplimiento con una valoración de 2,7 sobre 6 puntos.

Usabilidad y Navegabilidad

A partir de la observación se pudo constatar que el curso posee mapas de navegación, barras de situación, ayudas y herramientas de apoyo al estudiante. Igualmente, la pertinencia del diseño instruccional se realizó mediante la aplicación de un instrumento elaborado por SEDUCLA, en el cual se incluyen diferentes indicadores y estándares de calidad de la enseñanza en entornos virtuales. Sin embargo, no se encuentra la documentación referente a la organización y diseño del curso que facilite su navegación (características del entorno: intuitivo, fácil de utilizar, mapas de navegación, barras de situación, ayudas, herramientas de apoyo). Asimismo, no se ponen en práctica los resultados de la aplicación del test de usabilidad. De acuerdo a lo expuesto, se cumple con $60 \%$ de los criterios sugeridos en el

Calidad de un curso virtual de e-learning en ciencias de la salud Judith I Francisco Pérez, Judith J. Maldonado-Rivera, Manuel Ramón Blanco

DOI: http://dx.doi.org/10.22458/caes.v8i1.1774

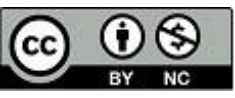

Artículo protegido por licencia Creative Commons 
Modelo CALED para la subárea usabilidad y navegabilidad que corresponde a una valoración de 1.8 puntos sobre 3 .

Mantenimiento

La Dirección de Telecomunicaciones y la Comisión de Informática de la UCLA, al momento de la evaluación, se encontraban trabajando para garantizar la escalabilidad de los equipos y programas informáticos. No se dispone de documentación oficial que evidencie que las tecnologías de hardware utilizadas posibilitan ser actualizadas en función de las necesidades y, que las tecnologías de software corresponden a estándares establecidos. Existe un equipo de desarrollo cualificado para el mantenimiento de los sistemas y su adecuación a las necesidades de la institución. Esta situación se muestra en el proceso de selección y evaluación para las contrataciones y concursos de oposición, en el cual, estas personas son evaluadas por un jurado designado por el Consejo Universitario, atendiendo al perfil establecido en el manual de cargos de la Oficina de Planificación del Sector Universitario (OPSU, s/f). Por otra parte, existen contratos de mantenimiento técnico de los sistemas de terceros y se dispone de licencias que permiten la actualización de los mismos. En esta subárea se cumple en un $75 \%$ con los estándares recomendados por el Modelo CALED, con una valoración 4.5 puntos sobre 6 .

La información antes presentada se resume en la Tabla № 1 que se presenta a continuación: 


\section{Tabla 1. Valoración de Estándares del Curso Virtual en el Área Tecnología.}

\begin{tabular}{llcl}
\hline \multirow{2}{*}{ Subáreas } & \multicolumn{2}{c}{ Valoración Parcial } & $\begin{array}{c}\text { Valoración } \\
\text { Total }\end{array}$ \\
\cline { 2 - 3 } Infraestructura Tecnológica & VP & $\%$ & $18 / 30$ \\
Disponibilidad, rendimiento y capacidad & 1.7 & 57 & \\
Seguridad y Privacidad & 4.3 & 51.25 & \\
Accesibilidad & 3 & 45 & \\
Usabilidad y Navegabilidad & 2.7 & 60 & 75 \\
Mantenimiento & 1.8 & & \\
Total & 4.5 & 18 & \\
\hline
\end{tabular}

Fuente: Formulario aplicado basado en la Guía de evaluación para cursos virtuales de formación continua CALED.

Autores: Francisco, J., Maldonado, J. y Blanco, M.

\section{Área: Formación.}

\section{Equipo Docente}

El equipo docente posee capacitación pedagógica y técnica antes del desarrollo del proceso formativo. La mayoría de los facilitadores posee título de Doctor en Tecnología Instruccional y Educación a Distancia, o maestría en el área. El apoyo pedagógico, en caso de ser necesario, está bajo la responsabilidad del Coordinador del Programa. Aunque no existe un documento, donde se informe a los participantes de dicha disponibilidad permanente, existe un equipo de apoyo tecnológico que atiende solicitudes a través de varias cuentas twitter: @soporteseducla, @seducla_informa y @seducla20. De igual manera, páginas de soporte usuario en www.facebook.com y números telefónicos para contactos directos. Con base en lo 
expuesto, se cumple en un $87,5 \%$ con los criterios establecidos en el modelo CALED que equivale a 7 puntos sobre 8 .

\section{Alumnos}

Los alumnos reciben un curso de formación técnica para el uso de los medios tecnológicos disponibles en el entorno de aprendizaje. Este curso tiene una duración de 96 horas y está diseñado con los mismos criterios del curso evaluado. En concordancia con esta información, se cumple plenamente con $100 \%$ de los criterios recomendados por el modelo CALED para la subárea correspondiente. La valoración es equivalente a un puntaje de 7 puntos sobre 7 . La información antes analizada se resume en la Tabla № 2, presentada a continuación.

\section{Tabla 2. Valoración de Estándares del Curso Virtual en el Área Formación}

\begin{tabular}{lccc}
\hline \multirow{2}{*}{ Subáreas } & \multicolumn{2}{c}{ Valoración Parcial } & \multirow{2}{*}{ Valoración Total } \\
\cline { 2 - 3 } & VP & $\%$ & \\
\hline Equipo docente & 7 & 87.5 & \\
Alumnos & 7 & 100 & $14 / 15$ \\
Total & 14 & & \\
\hline
\end{tabular}

Fuente: Formulario aplicado basado en la Guía de evaluación para cursos virtuales de formación continua CALED.

Autores: Francisco, J., Maldonado, J., y Blanco, M.

\section{Área: Diseño Instruccional}

Orientaciones Generales

El diseño instruccional del curso contiene orientaciones claras y precisas. Los participantes disponen desde el inicio, de un calendario académico con todas las actividades y fechas de 
interés. Asimismo, reciben la información general necesaria, tal como objetivos, metodología y sistema de atención. Desde el inicio del curso el participante conoce los medios de comunicación disponibles. Según lo señalado, se cumple plenamente con $100 \%$ de los criterios establecidos para esta subárea.

\section{Objetivos}

Los objetivos del curso están declarados de manera explícita en el diseño instruccional y aparecen, desde el inicio, destacados en el entorno de aprendizaje, por lo que se afirma el cumplimento del $100 \%$ de los criterios establecidos para esta subárea.

\section{Contenidos}

Los contenidos constan en la planificación del programa y en la guía didáctica del curso. Las unidades de aprendizaje son adecuadas y están directamente relacionadas con los recursos y actividades previstas, de manera que se promueve la concreción de lo aprendido y el desarrollo de la creatividad. Esto se apreció directamente en el entorno de aprendizaje virtual evaluado.

En este mismo sentido, los contenidos se presentan en unidades fácilmente manejables, y se proponen diferentes estrategias de aprendizaje, tales como: foros de discusión; elaboración de propuestas y ensayos; comparación y relación de términos, entre otros. No obstante, no se ha previsto ni se dispone de licencias para la publicación de contenidos, tampoco se han considerado medios alternativos de publicación de documentos, con el fin de entregar a los alumnos que no tienen acceso permanente a Internet o tienen conexiones de baja velocidad. Además, no se evidenciaron documentos que certifiquen que los contenidos se corresponden con los objetivos planteados. De acuerdo con los datos, se observa un bajo cumplimiento de 
los estándares de calidad establecidos, lo cual corresponde al 25\% con una valoración de 3,5 puntos sobre 14 .

\section{Interacción}

El entorno de aprendizaje garantiza la comunicación entre los diferentes actores del proceso formativo mediante el uso de diversas herramientas, tomando en cuenta el contexto y los objetivos perseguidos. Se diseñan actividades que fomentan la comunicación, el trabajo colaborativo y el intercambio entre los implicados en el proceso enseñanza aprendizaje. Asimismo, otras que promueven la interacción de los alumnos con los contenidos. Se utiliza la mensajería interna, el correo electrónico y el chat para la comunicación privada. A través de la red social de aprendizaje se fomentan las relaciones entre los implicados en el proceso formativo y se publican las reglas de "convivencia" en red que regirán el curso virtual. De acuerdo con lo señalado, la subárea "interacción" cumple con $100 \%$ de los estándares correspondientes.

Seguimiento y Tutoría.

La plataforma empleada posibilita el seguimiento y monitoreo permanente de las actividades desarrolladas por cada participante en el proceso formativo. Se dispone de estadísticas de navegación en forma inmediata; se identifican los contenidos revisados y las actividades virtuales realizadas por los alumnos; y, se conocen y valoran las interacciones efectuadas. Por otra parte, aunque en el diseño instruccional se señala que se proporcionará tutoría permanente a través del foro de discusión y foro de dudas, no existe ni se publica un plan de tutoría como tal, quedando el mismo a criterio de cada facilitador. Por tanto, no existe evidencias del plan de tutoría y su evaluación.

Calidad de un curso virtual de e-learning en ciencias de la salud Judith I Francisco Pérez, Judith J. Maldonado-Rivera, Manuel Ramón Blanco

DOI: http://dx.doi.org/10.22458/caes.v8i1.1774

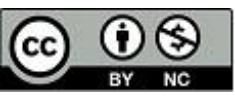

Artículo protegido por licencia Creative Commons 
Un aspecto a destacar es que, aunque en el Reglamento de EaD de la UCLA (UCLA, 2009) se establece el tiempo de respuesta máximo para atender las dudas de los participantes de los cursos virtuales, no existe un plan diseñado y aplicado por los responsables del curso para determinar si los facilitadores dieron respuesta a los estudiantes de manera oportuna. Algo positivo es que, en el entorno de aprendizaje, se lleva a cabo un proceso de retroalimentación interesante; se emplean diferentes herramientas para la tutoría en línea, como: foros, mensajería interna y correo electrónico; y, se publican anuncios para la tutoría presencial. Según la información recabada, se cumple satisfactoriamente con $77,5 \%$ de los estándares establecidos lo que equivale a una valoración de 4,65 puntos sobre 6 .

\section{Evaluación}

En general, el curso dispone de un plan de evaluación continua. El aula virtual cuenta con un sistema fiable para el registro de calificaciones, no susceptible de modificación por los estudiantes y con acceso a sus propias notas. El plan de evaluación considera las competencias a alcanzar mediante el trabajo individual y colaborativo de los estudiantes, asimismo, contempla pruebas de autoevaluación que posibilitan al alumno monitorear su evolución y grado de comprensión. Sin embargo, una debilidad es que no se garantiza la identidad de los estudiantes que completan exámenes online ni la confidencialidad de los datos personales. Tampoco se dispone de sistemas de evaluación alternativos para los participantes que no tienen acceso permanente a Internet. De acuerdo con los resultados observados, el curso virtual cumple con $75 \%$ de los estándares establecidos para la subárea "evaluación" con una calificación de 9 sobre 12 puntos.

A continuación, se presenta la Tabla $N^{\circ} 3$, donde se resumen los resultados del área Diseño Instruccional.

Calidad de un curso virtual de e-learning en ciencias de la salud Judith I Francisco Pérez, Judith J. Maldonado-Rivera, Manuel Ramón Blanco

DOI: http://dx.doi.org/10.22458/caes.v8i1.1774 


\section{Tabla 3. Valoración de Estándares del Curso Virtual en el Área Diseño Instruccional}

\begin{tabular}{lccc}
\hline & \multicolumn{2}{c}{ Valoración Parcial } & \multirow{2}{*}{ Valoración Total } \\
\cline { 2 - 3 } Subáreas & VP & $\%$ & \\
\hline Orientaciones generales del curso & 2 & 100 & \\
Objetivos & 5 & 100 & $30.1 / 45$ \\
Contenidos & 3.5 & 25 & \\
Interacción & 6 & 100 & \\
Seguimiento y tutoría & 4.6 & 77.5 & \\
Evaluación & 9 & 75 & \\
Total & 30.1 & & \\
\hline
\end{tabular}

Fuente: Formulario aplicado, basado en la guía de evaluación para cursos virtuales de formación continua CALED.

Autores: Francisco, J., Maldonado, J. y Blanco, M.

\section{Área: Servicios y soporte.}

Servicios de Información

Los participantes no disponen de un sitio web donde repose la información relacionada con la oferta del curso virtual, tal como, programa académico, mecanismos de admisión y matrícula, y fecha de inicio. La coordinación del sistema de educación a distancia del decanato establece sus propios criterios para la selección de los docentes que participarán en el programa de formación, pero estos criterios no son publicados para los interesados. Por otra parte, no existen listas de preguntas frecuentes para responder las dudas habituales que pudieran presentarse. 
En el entorno de aprendizaje se observa, que los estudiantes que se matriculan reciben con suficiente antelación la información referente a los requisitos tecnológicos y las condiciones de uso del sistema; mas no del sistema de privacidad de datos académicos y personales. De acuerdo con los hallazgos encontrados, se cumple en un $35 \%$ con los estándares establecidos que corresponde a 1,75 sobre 5 puntos.

\section{Atención al Alumno}

La coordinación del curso garantiza un servicio efectivo de atención al alumno. Se proporciona soporte técnico en línea a través de los siguientes números telefónicos: 58+251259-1530 Ext. 1530, 58+251-259-3914 Ext. 3914; así como, a través de las siguientes cuentas Twitter: @soporteseducla, @seducla_informa y @seducla20. Se dispone de servicios virtuales las 24 horas y se puede contar con tutores para asesoría asincrónica durante toda la semana. Esta información se evidencia en las orientaciones publicadas para el período de inducción en el entorno de aprendizaje del curso. De acuerdo con lo señalado, se cumple con $100 \%$ de los estándares recomendados que corresponde a 5 puntos sobre 5 . Estos datos analizados se resumen en la Tabla $N^{\circ} 4$, presentada a continuación.

Tabla 4. Valoración de Estándares del Curso Virtual en el Área Servicios y Soporte.

\begin{tabular}{lccc}
\hline Subáreas & \multicolumn{2}{c}{ Valoración Parcial } & \multirow{2}{*}{ Valoración Total } \\
\cline { 2 - 3 } & VP & $\%$ & \\
\hline Servicios de información & 1.75 & 35 & \\
Atención al alumno & 5 & 100 & $6.75 / 10$ \\
Total & 6.75 & &
\end{tabular}

Fuente: Formulario aplicado, basado en la guía de evaluación para cursos virtuales de formación continua CALED.

Autores: Francisco, J., Maldonado, J. y Blanco, M.

Calidad de un curso virtual de e-learning en ciencias de la salud Judith I Francisco Pérez, Judith J. Maldonado-Rivera, Manuel Ramón Blanco DOI: http://dx.doi.org/10.22458/caes.v8i1.1774 


\section{DISCUSIÓN DE RESULTADOS}

El curso tiene una valoración de 18 sobre 30 puntos en Calidad Tecnológica. Se desconoce el perfil tecnológico de los alumnos y, si las tecnologías disponibles son suficientes y adecuadas para el desarrollo del programa de formación. No se ponen en práctica los resultados de la aplicación del test de usabilidad. Tampoco existen planes de contingencia ante problemas técnicos y de previsión de tolerancia a fallos; ni la documentación referente a la organización y diseño del curso que facilite su navegación.

Asimismo, no se llevan registros que certifiquen que el curso fue migrado de un entorno experimental a otro formal, habiendo cumplido con todos los requerimientos y estándares. No hay documentación que evidencie que el hardware utilizado puede ser actualizado en función de las necesidades y que el software corresponde a estándares establecidos. Por estas razones, es importante fortalecer los estándares de esta área, tomando en cuenta que la calidad de la educación virtual implica disponer de tecnologías pertinentes, actualizadas y adecuadas (Aguilar y Bautista, 2015), y que el éxito de esta modalidad depende, entre otros aspectos, del uso y aplicabilidad que la institución haga de los recursos tecnológicos (GarcíaAretio, 2016).

En el área Formación hubo cumplimiento satisfactorio de 14 puntos sobre 15, justificada en el nivel de formación de los facilitadores y la asesoría disponible a través del entorno virtual. Esta fortaleza puede ayudar a la cohesión pedagógica que debe tener un sistema de educación bajo esta modalidad (Ardila-Rodríguez, 2011). No obstante, se requiere diseñar y publicar un documento informativo relacionado con el apoyo pedagógico que brinda la coordinación del curso a todos los participantes.

Calidad de un curso virtual de e-learning en ciencias de la salud Judith I Francisco Pérez, Judith J. Maldonado-Rivera, Manuel Ramón Blanco

DOI: http://dx.doi.org/10.22458/caes.v8i1.1774

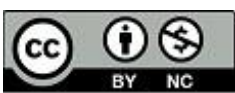

Artículo protegido por licencia Creative Commons 
Se encontró un cumplimiento satisfactorio en la mayoría de las subáreas e indicadores del Diseño Instruccional, alcanzando una valoración de 30.1 puntos sobre 45 . Los participantes son informados acerca las actividades, fechas de interés, objetivos, metodología y sistema de atención del curso. Igualmente, el entorno de aprendizaje garantiza la comunicación entre los diferentes actores del proceso formativo. Se diseñan actividades que fomentan la comunicación, el trabajo colaborativo y el intercambio entre participantes y docentes. Se utiliza la mensajería interna, el correo electrónico y el chat para la comunicación privada, y se publican las reglas de "convivencia" en red que regirán el curso virtual. Sin embargo, es necesario publicar un plan de tutoría para docentes y estudiantes, así como, fortalecer el uso de videoconferencias y establecer medios para garantizar la identidad de los participantes y la confiabilidad de sus datos personales. Además, se requiere sistematizar las evidencias del proceso de evaluación del curso antes de autorizar su implementación en el entorno formal.

En el área de servicios y soporte la valoración fue satisfactoria, con 6.75 puntos sobre 10. Hace falta un plan de comunicación sobre la oferta de cursos y elaborar listas de preguntas frecuentes para aclarar las dudas relacionadas con la oferta y desarrollo del curso virtual. Asimismo, incluir como parte de las orientaciones de la semana de inducción, información referida al sistema de privacidad de los datos académicos y personales.

La UCLA ha realizado un esfuerzo importante para promover la formación docente en elearning, así como para propiciar la autoevaluación del curso de educación continua en modalidad virtual. Este es el primer paso para corregir debilidades, y en el futuro alcanzar estándares que le permitan posicionarse como un centro de calidad de reconocida eficiencia y elevado nivel de formación (Francisco, 2012). Todo ello, considerando que son los docentes quienes diseñan y producen los materiales para promover el diálogo didáctico, generando aprendizaje individual, cooperativo y colaborativo (García Aretio, 2012). No

Calidad de un curso virtual de e-learning en ciencias de la salud Judith I Francisco Pérez, Judith J. Maldonado-Rivera, Manuel Ramón Blanco DOI: http://dx.doi.org/10.22458/caes.v8i1.1774

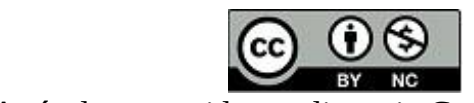


obstante, se requiere de un plan de fortalecimiento que permita responder a las exigencias tecnológicas de la modalidad y asegure la disponibilidad de entornos virtuales adecuados, que ofrezcan oportunamente todas las actividades y recursos requeridos para la autogestión del conocimiento y la calidad de formación (Ávila y Barragán, 2015).

\section{CONCLUSIONES}

La mayoría de los requerimientos tecnológicos y procesos requeridos por las distintas áreas y subáreas del modelo CALED, para garantizar la calidad del curso virtual, existen o son realizados por las diferentes instancias responsables, sin embargo, en muchos casos no se acompañan de registros, formatos e instrumentos que sirvan de evidencia al momento de la evaluación.

El curso no está soportado en un sistema que le permita solucionar los problemas generados por fallos de energía eléctrica, la cual es suplida por una empresa servidora externa a la universidad. Es decir, se carece de respaldos alojados en servidores externos para que el proceso educativo en línea tenga continuidad.

La institución dispone de profesionales en el área tecnológica que apoyan el proceso de capacitación de los docentes, sin embargo, no se conoce si éstos son adecuados o suficientes para apoyar el proceso de formación al que están comprometidos a participar.

Existen debilidades relacionadas con la verificación de la identidad del estudiante en las evaluaciones o pruebas del curso, comunes a cualquier sistema de educación virtual.

Calidad de un curso virtual de e-learning en ciencias de la salud Judith I Francisco Pérez, Judith J. Maldonado-Rivera, Manuel Ramón Blanco

DOI: http://dx.doi.org/10.22458/caes.v8i1.1774

\section{(c) (1) (3)}




\section{RECOMENDACIONES}

- A las Direcciones de Planificación, Finanzas y Compras de la UCLA, que incorporen en sus planes operativos el fortalecimiento de la infraestructura tecnológica de los diferentes decanatos, aumentando el número de equipos de acuerdo con las necesidades de aplicación de tecnologías en la enseñanza, tal como lo exigen las políticas académicas de la institución.

- A la Dirección de Telecomunicaciones: elaborar y tramitar la aprobación del manual de procedimientos que respalde todo lo relacionado con la seguridad y privacidad de la información manejada y generada dentro de los entornos virtuales.

- A las Direcciones de Telecomunicaciones e Informática de la UCLA, que coordinen la elaboración y aprobación de los formatos requeridos para certificar que el hardware puede ser actualizado en función de las necesidades y, que el software corresponde a estándares establecidos.

- Al SEDUCLA:

Promover el diseño y aprobación de protocolos, pautas, procedimientos y planes de contingencia que permitan prever y solucionar las eventualidades eléctricas sin afectar el desarrollo de los cursos.

Implementar un instrumento de evaluación basado en criterios de accesibilidad y usabilidad, para evidenciar que los cursos cumplen con los estándares requeridos para ser aprobados y migrados al entorno formal del sistema.

Elaborar un manual de normas y procedimientos con funciones y responsabilidades del personal, en concordancia con las funciones establecidas en el manual de cargos de la OPSU, y alineadas con su misión y visión. En ese mismo manual, hacer constar el procedimiento para la administración de las claves de acceso, acorde a la legislación

Calidad de un curso virtual de e-learning en ciencias de la salud Judith I Francisco Pérez, Judith J. Maldonado-Rivera, Manuel Ramón Blanco

DOI: http://dx.doi.org/10.22458/caes.v8i1.1774

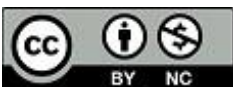

Artículo protegido por licencia Creative Commons 
vigente en materia de seguridad y custodia de datos personales. Además, garantizar un sistema de respaldo de la información y disponer de enlaces redundantes.

- A la Coordinación de Educación a Distancia del Decanato de Ciencias de la Salud: Implementar un instrumento de certificación del diseño instruccional de los cursos, para verificar la coherencia y la correspondencia de todos los componentes desde el punto de vista pedagógico.

Disponer de un portal web con la información sobre la oferta de los cursos, programas, condiciones de selección, cronogramas, entre otros.

- A la Coordinación Pedagógica del curso:

Incorporar un plan de tutoría y evaluación, que sirva de acompañamiento al estudiante ayudándolo a cumplir con los objetivos planificados.

Actualizar las pruebas de análisis de la audiencia para identificar la disponibilidad, naturaleza de equipos y programas informáticos; así como, la velocidad de conexiones a internet.

\section{REFERENCIAS}

Aguilar, R. M. y Bautista, M. J. (2015). Perfiles docentes y excelencia: Un estudio en la Universidad Técnica Particular de Loja, Ecuador. Revista Iberoamericana de Educación a Distancia, 18(2), 225-250. Recuperado de: http://revistas.uned.es/index.php/ried/article/view/13920/ Consultado el 10 de junio de 2016

Ardila-Rodríguez, M. (2011). Indicadores de calidad de las plataformas educativas digitales. Educación y Educadores, 14(1), 189-206. Recuperado de: http://www.redalyc.org/pdf/834/83418921011.pdf/ Consultado el 08 de junio de 2016

Avila, C. y Barragán, A. (2015). La calidad en los ambientes virtuales de aprendizaje. Una adaptación de categorías e indicadores para aplicar a los programas a distancia del contexto mexicano. Seminario La calidad de la educación superior no presencial. Universidad Autónoma de México. Recuperado de: 
http://reposital.cuaed.unam.mx:8080/jspui/bitstream/123456789/4517/1/La\%20calidad \%20en\%20los\%20ambientes\%20virtuales\%20de\%20aprendizaje\%20Avila\%20y\%20B arragan.pdf/ Consultado el 15 de marzo de 2016

Cabero-Almenara, J. (2016). La educación a distancia como estrategia de inclusión social y educativa. Revista mexicana de bachillerato a distancia, (15), 138-147. Recuperado de: $\quad$ http://bdistancia.ecoesad.org.mx/?articulo=la-educacion-a-distancia-comoestrategia-de-inclusion-social-y-educativa/ Consultado el 30 de noviembre de 2016

Francisco, J. (2012). Calidad en los entornos virtuales de aprendizaje. Revista Científica Compendium, 29(15), 97-107. Recuperado de: http://www.redalyc.org/pdf/880/88028701006.pdf/ Consutado el 03 de marzo de 2016

García Aretio, L. (20/06/2016). La calidad de la educación a distancia es, al menos, similar a la de la educación presencial $(16,12)$. Contextos universitarios mediados. (ISSN: 2340552X). Recuperado de https://aretio.hypotheses.org/2176/ Consutado el 03 de noviembre de 2016

García Aretio, L. (23/09/2013). MOOC. ¿Son EaD, igual que el e-learning? $(13,18)$.Contextos universitarios mediados. (ISSN: 2340-552X). Recuperado de http://aretio.hypotheses.org/736/ Consutado el 06 de abril de 2016

García, Aretio, L. (2012). El diálogo didáctico mediado en Educación a Distancia. Recuperado de http://aretio.hypotheses.org/380 Consultado el 30 de noviembre de 2016

García, Aretio, L. (1994). Educación a distancia hoy. Madrid, España: UNED

García, A., Guerrero, R. S. y Granados, J. M. (2015). Buenas prácticas en los entornos virtuales de enseñanza-aprendizaje. Revista Cubana de Educación Superior,34(3), 7688. Recuperado de http://scielo.sld.cu/scielo.php?script=sci_arttext\&pid=S025743142015000300006\&lng=es\&tIng=es\& Consultado el 15 de mayo de 2016

Hernández, R., Fernández, C. y Baptista, P. (2014). Metodología de la investigación. México, DF, México: McGraw-Hill/Interamericana Editores.

Oficina de Planificación del Sector Universitario de Venezuela. (s/f). Manual de Cargos. (Documento en línea). Recuperado de:

Calidad de un curso virtual de e-learning en ciencias de la salud Judith I Francisco Pérez, Judith J. Maldonado-Rivera, Manuel Ramón Blanco

DOI: http://dx.doi.org/10.22458/caes.v8i1.1774

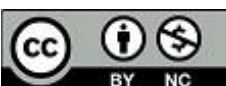

Artículo protegido por licencia Creative Commons 
http://www.ula.ve/personal/varios_opsu/Manual_Cargos.html/ Consultado el 03 de marzo de 2016

Universidad Centroccidental Lisandro Alvarado. (2009a). Reglamento y Procedimientos de la Dirección de Telecomunicaciones de la UCLA. (Documento en línea). Recuperado de: http://www.ucla.edu.ve/secretaria/Gacetas/GACETAS/GACETA\%20109/-

Reglamento\%20y\%20Procedimientos\%20de\%20TELECOMUNICACIONES.pdf/ Consultado el 07 de marzo de 2016

Universidad Centroccidental Lisandro Alvarado. (2009b). Reglamento de Educación a Distancia. Universidad Centroccidental Lisandro Alvarado-UCLA. (Documento en línea).

Recuperado dehttp://www.ucla.edu.ve/secretaria/Gacetas/GACETAS/GACETA\%20119/Reglament 0\%20de\%20la\%20Educacion\%20a\%20Distancia.pdf/ Consultado el 10 de marzo de 2016

Rubio, M., Morocho, M., Torres, J., Maldonado, J., Alejandro, J. \& Ramírez, I. (2009). Guía de Evaluación para Cursos Virtuales de Formación Continua. Instituto Latinoamericano y del Caribe de Calidad en Educación Superior a Distancia. UTPL. Loja-Ecuador.

Nuñez, M. (2017). Aprendizaje instantáneo. Observatorio de innovación educativa. Tecnológico de Monterrey. Disponible en https://observatorio.itesm.mx/edu-bitsblog/2017/4/24/aprendizaje-instantneo/ Consultado el 30 de marzo de 2017 